\title{
Correlation between Cognitive Functions and Activity of Daily Living among Post-Stroke Patients
}

\author{
Kurniawan Prakoso, ${ }^{1}$ Vitriana, ${ }^{2}$ Anam Ong ${ }^{3}$ \\ ${ }^{1}$ Faculty of Medicine Universitas Padjadjaran, ${ }^{2}$ Department of Physical Medicine and Rehabilition \\ Faculty of Medicine Universitas Padjadjaran/Dr. Hasan Sadikin General Hospital Bandung, \\ ${ }^{3}$ Department of Neurology Faculty of Medicine Universitas Padjadjaran/Dr. Hasan Sadikin \\ General Hospital Bandung
}

\begin{abstract}
Background: Cognitive impairment is one of the most common post-stroke complications; however, neither patients nor health professionals are often aware of this complication. The impact of cognitive impairment on quality of life is reflected through basic activity daily living (bADL) and instrumental activity daily living (IADL). Prior studies concerning the correlation between cognitive impairment and activity daily living has shown contradictive results. This study was conducted in order to analyze the correlation between the cognitive functions and activity daily living in post stroke patients at Dr. Hasan Sadikin General Hospital.

Methods: This cross-sectional study was carried out to 23 post-stroke patients from September-November 2015. Samples were collected through consecutive sampling at Dr. Hasan Sadikin General Hospital. Mini Mental State Examination (MMSE) was used to assess the cognitive functions and Lawton and Brody Scale to assess both bADL and IADL. Spearman correlation was selected to analyze the existing correlation between each cognitive domain and activity daily living.

Results: Spearman statistical correlation showed an insignificant correlation between the cognitive functions and bADL ( $\mathrm{r} 2=0.181, \mathrm{p}=0.408)$ and a significant correlation with IADL was obtained ( $2=0.517$, $\mathrm{p}=0.03)$. The only cognitive domain positively correlated with IADL was orientation to time and verbal recall. Conclusions: There is a correlation between cognitive functions and IADL among post-stroke patients at Dr. Hasan Sadikin General Hospital. [AMJ.2016;3(3):329-33]
\end{abstract}

Keywords: Activity of daily living, cognitive function, post stroke

\section{Introduction}

Stroke is one of a cerebrovascular diseases defined as a clinical syndrome consisting of rapidly developing clinical signs of focal (or global in case of coma) disturbance of cerebral functions that lasts more than 24 hours or leading to death with no apparent cause other than a vascular origin. ${ }^{1}$ Even though there is a decline in stroke mortality in America, it is still the fourth leading cause of death there. ${ }^{2}$ Stroke has many complications that may lead to neurological disorders, infections of several organs, immobility, thromboembolism, pain, and psychological disorders. ${ }^{3}$ Unfortunately, not all stroke patients and health professionals are aware of the impact induced by all those complications, especially the insidious complications such as cognitive impairment which may not be life threatening but will result in diminished quality of life, depicted by a lower score in basic and/or instrumental daily activities..$^{3-4}$

There are two different types of daily activities, namely, basic activity of daily living (bADL) and instrumental activity of daiy living (IADL). Basic activitiy of daily living consists of several activities responsible for self care. The components of bADL are bathing, toileting, dressing, feeding, functional mobility, personal device care, personal hygiene, grooming, sexual activity, and toilet hygiene..$^{5}$ On the other hand, IADL consists of several activities

Correspondence: Kurniawan Prakoso, Faculty of Medicine, Universitas Padjadjaran, Jalan Raya Bandung-Sumedang Km.21, Jatinangor, Sumedang, Indonesia, Phone: +6281320325048 Email: kurniawan_prakoso94@yahoo.co.id 
that are responsible for living independently in social life, such as care for others, care for pets, child rearing, communication management, community mobility, financial management, health management and maintenance, meal preparation and clean-up, religious observation, safety procedures and emergency responses, and shopping. ${ }^{5}$

Some of the studies conducted previously on the relation between cognitive impairment and bADL or IADL following stroke have shown contradictive results. A study by Akbari et al. ${ }^{6}$ concluded that there was no correlation between thinking process and bADL measured by Barthel Index (BI), but the study of Ghosal et al. ${ }^{7}$ had discovered an opposite result. The description on the correlation between the cognitive functions and both bADL and IADL may be necessary for planning rehabilitation for post-stroke patients so that they have fulfilling quality of life. Ironically, there are not many studies about the correlation between cognitive functions and bADL or IADL in Indonesia. For that reason, this study was conducted to analyze the correlation between the cognitive functions and both bADL and IADL.

\section{Methods}

This study was conducted from September to November 2015 using cross-sectional study design. Samples were collected through consecutive sampling from all post-stroke patients who visited the neurology clinic at Dr. Hasan Sadikin General Hospital Bandung from September-November 2015. The samples were directly assessed for inclusions and exclusions criteria to minimize bias and confounding factors. Initial minimum sample size of 42 subjects was set before conducting this study. Patients who were aphasic, older than eighty five years old, or did not graduate from elementary school were not recruited in this study. ${ }^{7}$ After checking the patient's manual muscle testing (MMT) results, there were only 23 eligible respondents with MMT $\geq 3$ recruited as this study subjects. Those subjects would then be assessed for cognitive functions, bADL, and IADL.

The cognitive functions were evaluated using the Mini-Mental State Examination (MMSE) that has been applied to evaluate global cognitive functions for many years. The highest possible score of 30 can be obtained from eleven items of five cognitive domain (orientation, registration, attention and calculation, recall, and language). ${ }^{8}$ Activity daily living in this study was measured using M.P. Lawton and E.M. Brody Scale consisting of six items for bADL (toileting, feeding, dressing, grooming, mobility, and bathing) and eight items for IADL (using telephone, food prepration, shopping, house keeping, laundry, mode of transportation, responsibility for own medication, and handling finances). ${ }^{9}$ Each item of both bADL and IADL in Lawton score has 1 point. A total score of 6 and 8 will be obtained as a perfect score for bADL and IADL respectively.

Simple correlation statistical analysis was carried out to check the correlation between the cognitive functions and bADL and IADL. Since normality testing showed abnormal data distribution for both bADL and IADL, Spearman correlation was selected to check the correlation between the cognitive functions and bADL or IADL. Ethics approval was obtained from Dr. Hasan Sadikin General Hospital Ethics Committee.

\section{Results}

Table 1 describes the demographic characteristics of the subject. The average age of this study population was $58.26 \pm 2.658$ years and the median for the years of education is 12 years, which was above the minimum requirement for this study recruitment. The most frequent type of stroke occured in this study was the ischemic stroke. The mean cognitive functions based on the MMSE assessment was 23 .

After checking the data distribution using Saphiro-Wilk normality test, Spearman correlation test was used to analyze the correlation strength and significance between the cognitive functions and both bADL and IADL. The MMSE had a significant positive correlation with IADL ( $2=0.517, p=0.03$ ). On the contrary, the correlation between cognitive functions and bADL was insignificant ( $\mathrm{r} 2=$ $0.181, p=0.408$ ).

Results from further evaluation of the correlation between each cognitive function and IADL are listed in table 3 . Out of five cognitive domains, only two had significant correlations with IADL. Orientation to time had a stronger positive correlation with IADL ( $\mathrm{r} 2=0.517, \mathrm{p}=0.011$ ), while recall had a slightly weaker positive correlation with IADL ( $\mathrm{r} 2=0.424, \mathrm{p}=0.044$ ).

As the orientation to time and recall were the only cognitive domains that were correlated with IADL, further analysis regarding these two domain correlations with each IADL 
Table 1 Patient Characteristics

\begin{tabular}{lcc}
\hline \multicolumn{1}{c}{ Characteristics } & Mean (SD), or Median (Min-Max) & n \\
\hline Age (Years, SD) & $58.26(2.658)$ & 23 \\
Gender & & 9 \\
Male (n) & & 14 \\
Female (n) & $12(6-15)$ & 23 \\
Education (Years) & & \\
Type of stroke & & 18 \\
Ischemic (n) & $10(1-15)$ & 5 \\
Hemorrhagic (n) & $23(1.085)$ & 23 \\
Duration after stroke (Months) & $6(3-6)$ & 23 \\
Cognitive function (0-30, SD) & $5(0-8)$ & 23 \\
bADL (0-6) & & 23 \\
IADL (0-8)
\end{tabular}

Note : IADL = Instrumental Activity Daily Living; bADL = Basic Activity Daily Living

items was conducted. Out of 8 items of IADL, only using telephone, handling finances, and responsibility for own medication were significantly correlated with the orientation to time. Verbal recall was also significantly correlated with food preparation ( $\mathrm{r} 2=0.597$, $\mathrm{p}=0.003)$.

\section{Discussion}

Cognitive impairment is often discovered in stroke patients as a complication following the stroke. Prior descriptive study held in Korea ${ }^{10}$ revealed that out of 353 samples, 221 (62.6\%) were cognitively impaired. The other study discovered that the average MMSE score was 23 , indicating a mild cognitive impairment.11 This condition might lead to interference for post-stroke patients in doing their daily activities. ${ }^{12}$

Daily activities are divided into basic daily activities (bADL) and instrumental daily activites (IADL). Basic activity daily living (bADL) is essential for a person to get their basic daily needs. ${ }^{5}$ These activities need a good executive cognitive function in order to be carried out. ${ }^{13}$ Executive function is essential in planning and implementing basic daily activities, which, unfortunately, cannot be assessed using MMSE. ${ }^{5,13}$ There are many suitable instruments for assessing the executive function such as Modified Wisconsin Card Sorting Test, The Trail Making Test $\mathrm{A}$ and $\mathrm{B}$, the Stroop Interference Test, and Verbal Fluency Test; however, the translated and validated indonesian version of those instruments are not available yet. ${ }^{13}$ Hence,

Table 2 Cognitive Domain Correlation with IADL

\begin{tabular}{lcc}
\hline \multirow{2}{*}{ Cognitive Domain } & \multicolumn{2}{c}{ IADL } \\
\cline { 2 - 3 } & $\mathbf{~ r 2}$ & $\mathbf{p}$ \\
\hline Orientation to Time & $0.517^{*}$ & 0.011 \\
Orientation to Place & 0.342 & 0.110 \\
Registration & 0.261 & 0.229 \\
Atention and Calculation & 0.328 & 0.127 \\
Recall & $0.424^{*}$ & 0.044 \\
Language & 0.224 & 0.305 \\
\hline Note: ${ }^{*}$ Correlation is significant at the 0.01 level (2-tailed), IADL = Instrumental Activity Daily Living
\end{tabular}


Table 3 Correlation between IADL Items and both Orientation to Time and Recall

\begin{tabular}{lcccc}
\hline \multirow{2}{*}{ IADL items } & \multicolumn{2}{c}{ Orientation to Time } & \multicolumn{2}{c}{ Recall } \\
\cline { 2 - 5 } & $\mathbf{r} 2$ & $\mathbf{p}$ & $\mathbf{r 2}$ & $\mathbf{p}$ \\
\hline Using Telephone & $0.580^{* *}$ & 0.004 & 0.203 & 0.353 \\
Shopping & 0.239 & 0.271 & 0.146 & 0.499 \\
Food Preparation & 0.328 & 0.126 & $0.597^{* *}$ & 0.003 \\
House Keeping & 0.059 & 0.788 & 0.207 & 0.344 \\
Laundry & 0.100 & 0.650 & 0.294 & 0.174 \\
Mode of Transportation & 0.254 & 0.243 & 0.113 & 0.609 \\
Responsibility for Own Medication & $0.493^{*}$ & 0.017 & -0.008 & 0.971 \\
Handling Finances & $0.493^{*}$ & 0.017 & 0.214 & 0.326 \\
\hline Note: ${ }^{*}$ Correlation is significant at the 0.05 level $\left(2\right.$-tailed), ${ }^{* *}$ Correlation is significant at the 0.01 level (2-tailed), IADL \\
$=$ Instrumental Activity Daily Living; r $=$ Correlation strength from spearman correlation
\end{tabular}

MMSE is selected in this study to assess the cognitive functions since it is a tool capable of evaluating global cognitive functions, though superficially.

At the same time, bADL activities that are not as complex as IADL, may be correlated with the cognitive functions if the subjects assessed had a very high dependency. ${ }^{14}$ Thus, it is assumed that the insignificant relationship between bADL and cognitive functions in this study is resulted from the inappropriate use of instrument responsible, for evaluating cognitive functions and bADL total score average that shows little or no dependency.

Unlike bADL which consists of simple activities that require good motoric and executive functions, instrumental activity daily living (IADL) consists of more complex activities closely related to cognitive functions. ${ }^{15}$ These activities are needed for a person to adapt to their environment and social life. ${ }^{5}$ The activities presented in Lawton and Brody IADL Scale consist of ability to use telephone, shopping, food preparation, house keeping, laundry, mode of transportation, responsibility for own medications, and ability to handle finance. ${ }^{9}$ In this study, these activities were generally correlated with cognitive functions especially with orientation to time and verbal recall. This possibly might be due to the correlations between several activities of IADL, like using telephone, handling finance, and responsiblity for own medication with orientation to time. Food preparation was also significantly correlated with verbal recall. This results are coherent with a previous study claiming that using technology, in this case, using telephone and handling finance, correlates with memory function which, in this study, is reflected by the orientation to time and verbal recall. ${ }^{13}$ Orientation to time is also essential for a person to make a schedule, and in the case of drug consumption, to make a person responsible for his own medication.

The other four items in Lawton and Brody IADL may not correlate with the cognitive functions assessed by MMSE, due to the same reason, as the absence of correlation between ADL and cognitive functions in this study. Even though shopping, housekeeping, laundry, and mode of transportation are included as IADL activities due to their roles in a person's adaptation to his/her environment and social life, they are more likely to be influenced by motor function and executive function. ${ }^{13}$ It is clearly shown that these activities are not parts of complex activities which require memory function.

This study has several limitations due to the narrow time frame for data collection resulting in inadequate sample size compared to the size originally calculated of a minimum of 42 participants. Furthermore, the inability of MMSE for assessing executive cognitive functions which are supposed to be correlated with bADL may lead to underrated results of the study.

In conclusion, there is a correlation between the cognitive functions and IADL in post-stroke patients at Dr. Hasan Sadikin General Hospital Bandung. Thus, it is essential for post-stroke patients or, at the very least, health professionals, to be more concern about cognitive impairments as a prevalent complication following stroke due to its effect on the post-stroke quality of life. Rehabilitation on post-stroke patient should not only focus on motor functions that may be crucial for 
the patient to conduct basic daily activities but also on cognitive functions as they are essential for performing complex activities. This is important because the respondents of this study are on average, still in their productive age.

Further studies regarding the correlation between cognitive functions and activity daily living may be needed in order to further explore the involvement of each cognitive domain to every activity daily living items; hence, appropriate rehabilitation approaches can be given to support the post-stroke patients with cognitive impairment and dependency.

\section{References}

1. Aho $K$, Harmsen P, Hatano $S$, Maquardsen J, Smirnov VE, Strasser T. Cerebrovascular disease in the community: results of a WHO collaborative study. Bull World Health Organ. 1980;58(1):113-30.

2. Burke JF, Lisabeth LD, Brown DL, Reeves MJ, Morgenstern LB. Determining stroke's rank as a cause of death using multicause mortality data. Stroke. 2012;43(8):220711.

3. Pandian JD, Kaur A, Jyotsna R, Padma MV, Venkateswaralu K, Sukumaran S, et al. Complications in acute stroke in India (CAST-I): a multicenter study. J Stroke Cerebrovasc Dis. 2012;21(8):695-703.

4. Cumming TB, Brodtmann A, Darby D, Bernhardt J. The importance of cognition to quality of life after stroke. J Psychosom Res. 2014;77(5):374-9.

5. Buzaid A, Dodge MP, Handmacher L, Kiltz PJ. Activities of daily living evaluation and treatment in persons with multiple sclerosis. Phys Med Rehabil Clin N Am. 2013;24(4):629-38.

6. Akbari S, Lyden PD, Kamali M, Fahimi MA. Correlations among impairment, daily activities and thinking operations after stroke. NeuroRehabilitation. 2013;33(1):153-60.

7. Ghosal MK, Burman P, Singh V, Das S, Paul $\mathrm{N}$, Ray BK, et al. Correlates of functional outcome among stroke survivors in a developing country-a prospective community-based study from India.J Stroke Cerebrovasc Dis. 2014;23(10):2614-21.

8. Tatari F, Farnia V, Kazemi F. Mini Mental State Examination (MMSE) in first episode of psychosis. Iran J Psychiatry. 2011;6(4):158-160.

9. Pohjasvaara TI, Jokinen $H$, Ylikoski R, Kalska H, Mantyla R, Kaste M, et al. White matter lesions are related to impaired instrumental activities of daily living poststroke. J Stroke Cerebrovasc Dis. 2007;16(6):251-8.

10. Yu KH, Cho SJ, Oh MS, Jung S, Lee JH, Shin $\mathrm{JH}$, et al. Cognitive impairment evaluated with vascular cognitive impairment harmonization standards in a multicenter prospective stroke cohort in Korea. Stroke. 2013;44(3):786-8.

11. Pezzotti P, Scalmana S, Mastromattei A, Di. Lallo D. The accuracy of the MMSE in detecting cognitive impairment when administered by general practitioners: a prospective observational study. BMC Fam Pract. 2008;9:29-30.

12. Feigin VL, Forouzanfar MH, Krishnamurthi $\mathrm{R}$, Mensah GA, Connor M, Benett DA, et al. Global and regional burden of stroke during 1990-2010: findings from the Global Burden of Disease Study 2010. Lancet. 2014;383(9913):245-54.

13. Mograbi DC, Faria CdA, FIchman HC, Paradela EM, Lourenço RA. Relationship between activities of daily living and cognitive ability in a sample of older adults with heterogeneous educational level. Ann Indian Acad Neurol. 2014;17(1):71-6.

14. Helvk A-S, Høgseth LD, Bergh S, ŠaltytèBenth J, Kirkevold Ø, Selbæk G. A 36-month follow-up of decline in activities of daily living in individuals receiving domiciliary care. BMC Geriatr. 2015;15(47):1-13.

15. Haaland KY, Mutha PK, Rinehart JK, Daniels M, Cushnyr B, Adair JC. Relationship between arm usage and instrumental activities of daily living after unilateral stroke. Arch Phys Med Rehabil. 2012;93(11):1957-62. 